\title{
Jamming and Static Stress Transmission in Granular Materials
}

\author{
M. E. Cates \\ Dept. of Physics and Astronomy, University of Edinburgh \\ JCMB King's Buildings, Mayfield Road, Edinburgh EH9 3JZ, GB. \\ J. P. Wittmer \\ Départment de Physique des Matériaux, Université Lyon I $\&$ CNRS \\ 69622 Villeurbanne Cedex, France. \\ J.-P. Bouchaud, P. Claudin \\ Service de Physique de l'Etat Condensé, CEA \\ Ormes des Merisiers, 91191 Gif-sur-Yvette Cedex, France.
}

\begin{abstract}
We have recently developed some simple continuum models of static granular media which display "fragile" behaviour: they predict that the medium is unable to support certain types of infinitesimal load (which we call "incompatible" loads) without plastic rearrangement. We argue that a fragile description may be appropriate when the mechanical integrity of the medium arises adaptively, in response to a load, through an internal jamming process. We hypothesize that a network of force chains (or "granular skeleton") evolves until it can just support the applied load, at which point it comes to rest; it then remains intact so long as no incompatible load is applied. Our fragile models exhibits unusual mechanical responses involving hyperbolic equations for stress propagation along fixed characteristics through the material. These characteristics represent force chains; their arrangement expressly depends on the construction history. Thus, for example, we predict a large difference in
\end{abstract}


the stress pattern beneath two conical piles of sand, one poured from a point source and one created by sieving.

Granular materials are microscopically heterogenous. Despite this, it is natural to search for continuum models that can describe their static and dynamic behaviour. The problem of granular statics implicitly requires knowledge of the construction history of the medium. At the microscopic scale, the construction history determines exactly where every grain is, and how it has been deformed from its original shape. Given this information, the microscopic forces follow from the local contact mechanics. But such a microscopic description of granular materials is, in practical terms, impossible and unlikely to be a useful guide to their macroscopic behaviour. For example it is often assumed that if elasticity governs the local contact mechanics, the continuum behaviour of the assembly as a whole must be elastic. This may be unjustified: the physics of the granular assembly involves additional, strongly nonlinear physics - namely, whether each contact is actually present or not. If, as we believe, the contact network is an adaptive structure that has organized itself to support the specific load applied during construction, it may obey continuum equations quite unlike those of conventional elastic or elastoplastic media.

\section{INTRODUCTION}

In this paper we consider assemblies of cohesionless rough particles, whose rigidity is sufficient that individual particle deformations remain always small. Such assemblies are sometimes argued to be governed by the continuum mechanics of a Hookean elastic solid (perhaps with a very high modulus). But this implicitly assumes that each granular contact is capable equally of supporting tensile as compressive loads. For a cohesionless medium this is certainly untrue: cohesionless granular assemblies are therefore not elastic. [1] The 
question is not one of principle, but of degree - how important is the prohibition of tensile forces? This is not completely clear; some would argue [2] that it represents a negligible effect and that an elastic model remains basically sound, so long as the mean stresses in the material remain compressive everywhere. However, a fully elastic granular assembly would be one in which grains were, effectively, glued permanently to their neighbours on first contact. Because the packing is microscopically disordered, it is possible that, during subsequent loading a significant fraction of such contacts would become tensile, even if the load being applied remains everywhere compressive on average. If so, the absence of tensile forces is a major, even dominant, factor.

Notice that the absence of tensile forces is a distinct physical effect from the one addressed by most elastoplastic continuum theories of granular media (see, e.g., Ref. [3]). These are like elastic models, but they allow for the fact that the ratio of shear and normal forces at a contact cannot exceed a fixed value determined by a coefficient of static friction; this is usually assumed to translate to a similar condition on the bulk stress components acting across any plane. The resulting plasticity is similar to that arising in metals, for example, and not related to the prohibition of tensile forces: it applies equally for cohesive contacts. Of course, in applying such theories to cohesionless media one should assume that the mean stresses are everywhere compressive: however, as emphasized above, this constraint does not ensure that individual contact forces are all compressive as is actually required.

These considerations suggest a physically very different picture of granular media, already well developed and respected in the sphere of discrete modelling. [4] In this picture, nonlinear physics is dominant, and the contact network of grains is always liable to reorganize as loads are applied: it is an "adaptive structure". [5] The contact network defines a loadbearing "granular skeleton" shown in Fig. 1(a): this is often thought of as a network of "force chains", or roughly linear chains of strong particle contacts, alongside which the other grains play a relatively minor role in the transmission of stress. [5, 6] If these ideas are true, the continuum mechanics of the material has to be thought about afresh. Since this widely-accepted picture of force-chains implies a microscopically heterogeneous character of 
the contact network in the material, it is not necessarily obvious that a continuum description of it is possible at all. However, we have in recent years developed continuum models for granular materials which, we now believe, do capture some of the physics of force chains, and of their geometrical dependence on the construction history. This interpretation, which has evolved significantly beyond the empiricism of our early work, [7 9] is developed below.

The proposal that granular assemblies under gravity cannot properly be described by the ideas of conventional elastoplasticity has been opprobiously dismissed in some quarters: we stand accused of ignoring all that is 'long and widely known' among geotechnical engineers. [10] However, we are not the first to put forward such a subversive proposal. Indeed workers such as Trollope [11] and Harr [12] have long ago developed ideas of force transfer rules among discrete particles, not unrelated to our own approaches, which yield continuum equations quite unlike those of elastoplasticity. [13]16] More recently, dynamical hypoplastic continuum models have been developed [17] which, as explained by Gudehus [18 describe an 'anelastic behaviour without [the] elastic range, flow conditions and flow rules of elastoplasticity'. Our own models, though not explicitly dynamic, are similarly anelastic in a specific manner that we describe as "fragile".

In Section 1 l] below, we describe a generic "jamming" mechanism for the construction of a granular skeleton that, we argue, points toward fragile mechanical behaviour. This scenario is related, but not identical, to several other current ideas in the literature on granular media. [1, 6, 17, 19 25] These include the emergence of rigidity by successive buckling of force chains [21] and the concept of mechanical percolation. [17] In particular there is a strong link between fragile media and isostatic models of granular assemblies. [1,26] In an isostatic network, the requirement of force balance at the nodes is enough to determine all the forces acting, so these can be calculated without reference to a strain or displacement variable. Isostatic networks require a mean coordination number with a specific critical value $(z=2 d$ with $d$ the dimension of space). In this sense, isostatic contact networks are "exceptional", and may appear remote from real granular materials.

However, it is increasingly clear 23,25] that almost all disordered packings of frictionless 
spheres actually approach an isostatic state in the rigid particle limit. Since friction is ignored, there is still a missing link between this result and the physics of real granular media - a link provided by the concept of force chains, as we show below (Section IIB). More generally, the idea that the granular skeleton could engineer itself to maintain an isostatic or fragile state is closely connected with the concepts of self-organized criticality (SOC) 222] (see also Ref. [27]). The concepts provide a generic mechanism whereby an overdamped dynamical system under external forcing can come to rest at a marginally stable (critical) state. In the SOC scenario, this state is characterized by hierarchical (fractal) correlations and large noise effects (compare Fig. 1(a)). In this article we ignore these complications and describe only our minimalist, noise-free models of the granular skeleton; these represent, in effect, regular arrays of force chains. The effect of noise on the resulting continuum equations is of great interest, but these require a separate discussion, which is made elsewhere. 13, 14.

\section{COLLOIDS, JAMMING AND FRAGILE MATTER}

\section{A. Colloids}

We start by describing a simple model of jamming in a colloid, sheared between parallel plates. 16] This is the simplest plausible scenario in which an adaptive skeleton forms in response to an applied load; we believe it sheds much light on the related problem of dry granular media as discussed in Section [II below. We will first use it to illustrate some general ideas on the relationship between jamming and fragility.

Consider a concentrated colloidal suspension of hard particles, confined between parallel plates at fixed separation, to which a shear stress is applied (Fig. 1 (b) and 目 (a)). Above a certain threshold of stress, this system exhibits enters a regime of strong shear thickening; see, e.g., Ref. 28]. The effect can be observed in the kitchen, by stirring a concentrated suspension of corn-starch with a spoon. In fact, computer simulations suggest that, at least under certain idealized conditions, the material will jam completely and cease to flow, no matter 
how long the stress is maintained. 229 In these simulations, jamming apparently occurs because the particles form "force chains" [4] along the compressional direction (Fig. 11(b)). Even for spherical particles the lubrication films cannot prevent direct interparticle contacts; once these arise, an array or network of force chains can indeed support the shear stress indefinitely. (We ignore Brownian motion, here and below, as do the simulations; this could cause the jammed state to have finite lifetime.)

To model the jammed state, we start from a simple idealization of a force chain: a linear string of at least three rigid particles in point contact. Crucially, this chain can only support loads along its own axis (Fig.3 (a)): successive contacts must be collinear, with the forces

along the line of contacts, to prevent torques on particles within the chain. [19] Note that neither friction at the contacts, nor particle aspherity, can change this "longitudinal force" rule. (Particle deformability, however, does matter; see Section $\llbracket I I \mathrm{~g}$ below.)

As a minimal model of the jammed colloid, we therefore take an assembly of such force chains, characterized by a unique director (a headless unit vector) n, in a sea of "spectator" particles, and incompressible solvent. This is obviously oversimplified, for we ignore completely any interactions between chains, the deflections caused by weak interactions with the spectator particles, and the fact that there must be some spread in the orientation of the force chains themselves. Nonetheless, with these assumptions, in static equilibrium and with no body forces acting, the pressure tensor $p_{i j}$ (defined as $p_{i j}=-\sigma_{i j}$, with $\sigma_{i j}$ the usual stress tensor) must obey

$$
p_{i j}=P \delta_{i j}+\Lambda n_{i} n_{j}
$$

Here $P$ is an isotropic fluid pressure, and $\Lambda(>0)$ a compressive stress carried by the force chains.

\section{B. Jamming and Fragile Matter}

Eq. (四) defines a material that is mechanically very unusual. It permits static equilibrium only so long as the applied compression is along $\mathbf{n}$; while this remains true, incremental loads 
(an increase or decrease in stresses at fixed compression axis of the stress tensor) can be accommodated reversibly, by what is (at the particle contact scale) an elastic mechanism. But the material is certainly not an ordinary elastic body, for if instead one tries to shear the sample in a slightly different direction (causing a rotation of the principal stress axes) static equilibrium cannot be maintained without changing the director $\mathbf{n}$. Now, $\mathbf{n}$ describes the orientation of a set of force chains that pick their ways through a dense sea of spectator particles. Accordingly $\mathbf{n}$ cannot simply rotate; instead, the existing force chains must be abandoned and new ones created with a slightly different orientation. This entails dissipative, plastic, reorganization, as the particles start to move but then re-jam in a configuration that can support the new load. The entire contact network has to reconstruct itself to adapt to the new load conditions; within the model, this is true even if the compression direction is rotated only by an infinitesimal amount.

Our model jammed colloid is thus an idealized example of "fragile matter" : it can statically support applied shear stresses (within some range), but only by virtue of a self-organized internal structure, whose mechanical properties have evolved directly in response to the load itself. Its incremental response can be elastic only to compatible loads; incompatible loads (in this case, those of a different compression axis), even if small, will cause finite, plastic reorganizations. The inability to elastically support some infinitesimal loads is our chosen technical definition of the term "fragile". 16]

We argue that jamming may lead generically to mechanical fragility, at least in systems with overdamped internal dynamics. Such a system is likely to arrests as soon as it can support the external load; since the load is only just supported, one expects the state to be only marginally stable. Any incompatible perturbations then force rearrangement; this will leave the system in a newly jammed but (by the same argument) equally fragile state. This scenario is related, but not identical, to several other ideas in the literature. [17, 19,23] The link between jamming and fragility is schematically illustrated in Fig. 4 .

Now consider again the idealized jammed colloid of (Fig. 2 (a)). So far we allowed for an external stress field (imposed a the plates) but no body forces. What body forces can the 
system support without plastic rotation of the director? Various models are possible. One is to assume that Eq. (11) continues to apply, with $P(\mathbf{r})$ and $\Lambda(\mathbf{r})$ now varying in space. If $P$ is a simple fluid pressure, a localized body force can be supported only if it acts along n. Thus (as in a bulk fluid) no static Green function exists for a general body force. (Note that, since Eq. (11) is already written as a continuum equation, such a Green function would describes the response to a load that is localized in space but nonetheless acts on many particles in some mesoscopic neighbourhood.) For example, if the particles in Fig. 2 (a) were to become subject to a gravitational force along $y$, then the existing force chains could not sustain this but would reorganize. Applying the longitudinal force rule, the new shape is easily found to be a catenary, as realized by Hooke, [30] and emphasized by Edwards. [19] On the other hand, a general body force can be supported, in three dimensions, if there are several different orientations of force chain, possibly forming a network or "granular skeleton". [6, 17] A minimal model for this is:

$$
p_{i j}=\Lambda_{1} n_{i} n_{j}+\Lambda_{2} m_{i} m_{j}+\Lambda_{3} l_{i} l_{j}
$$

with $\mathbf{n}, \mathbf{m}, \mathbf{l}$ directors along three nonparallel populations of force chains; the $\Lambda$ 's are compressive pressures acting along these. Body forces cause $\Lambda_{1,2,3}$ to vary in space.

We can thus distinguish two levels of fragility, according to whether incompatible loads include localized body forces (bulk fragility, e.g. Eq. (11)), or are limited to forces acting at the boundary (boundary fragility, e.g. Eq. (2)). In disordered systems one should also distinguish between macro-fragile responses involving changes in the mean orientation of force chains, and the micro-fragile responses of individual contacts. We expect micro-fragility in granular materials (see Ref. [27]), although the models discussed here, which exclude randomness, are only macro-fragile; in practice the distinction may become blurred. In any case, these various types of fragility should not be associated too strongly with minimal models such as Eqs. (11,2). It is clear that many granular skeletons have a complex network structure where many more than three directions of force chains exist. Such a network may nonetheless be fragile. 
Fragility in fact requires any connected granular skeleton of force chains, obeying the longitudinal force rule (LFR), to have a mean coordination number $z=2 d$ with $d$ dimension of space (e.g. Fig. 2(b) in two dimensions). This coordination number describes the skeleton, rather than the medium as a whole; but otherwise it is the same rule as applies for packings of frictionless hard spheres. These also obey the LFR - not because of force chains, but because there is no friction. Regular packings of frictionless spheres (which show isostatic mechanics) have been studied in detail recently; [1,13,20] and Moukarzel has argued that disordered frictionless packings of hard spheres are also generically fragile [23] (see also Ref. 25]). These arguments appear to depend only on the LFR and the absence of tensile forces, so they should, if correct, equally apply to any granular skeleton that is made of force chains of three or more completely rigid particles.

\section{Fixed Principal Axis Model}

Returning to the simple model of Eq. (2), the chosen values of the three directors (two in $d=2$ ) clearly should depend on how the system came to be jammed (its "construction history"). If it jammed in response to a constant external stress, switched on suddenly at some earlier time, one can argue that the history is specified purely by the stress tensor itself. In this case, if one director points along the major compression axis then by symmetry any others should lie at rightangles to it (Fig. 22 (b)). Applying a similar argument to the intermediate axis leads to the ansatz that all three directors lie along principal stress axes; this is perhaps the simplest model in $d=3$. One version of this argument links force chains with the fabric tensor, [17] which is then taken coaxial with the stress. [6] (The fabric tensor is the second moment of the orientational distribution function for contacts and/or interparticle forces.)

With the ansatz of perpendicular directors as just described, Eq. (2) becomes a "fixed principle axes" (FPA) model. [8,9, 13] Although grossly oversimplified, this leads to nontrivial predictions for the jammed state in the colloidal problem, such as a constant ratio of the 
shear and normal stresses when these are varied in the jammed regime. Such constancy is indeed reported by Laun [28] in the regime of strong shear thickening; see Ref. [16].

\section{GRANULAR MATERIALS}

We believe that these simple ideas on jamming and fragility in colloids are equally relevant to cohesionless, dry granular media constructed from hard frictional particles. For although the formation of dry granular aggregates under gravity is not normally described in terms of jamming, it is a closely related process. Indeed, the filling of silos and the motion of a piston in a cylinder of grains both exhibit jamming and stick-slip phenomena associated with force chains; see Ref. [31]. And, just as in a jammed colloid, the mechanical integrity of a sandpile entirely disappears as soon as the load (in this case gravity) is removed.

In the granular context, a model like Eq. (2) can be interpreted by saying that a fragile granular skeleton of force chains is laid down at the time when particles are first buried at a free surface (see Fig. 5); so long as subsequent loadings are compatible with this structure, the skeleton will remain intact - if not grain by grain, then at least in its average properties. If in addition the skeleton is rectilinear (perpendicular directors) this forces the principal axes to maintain forever the orientation they had close to the free surface (FPA model). However, we do not insist on this last property and other models, which correspond to an oblique family of directors in Eq. (2), will be described below. [9, 16, 15]

In what follows we review in more detail the nature of our fragile models and the role they might play within a continuum mechanical description of granular media. We will mainly be concerned with the standard sandpile, which we define to be a conical pile, constructed by pouring cohesionless hard grains from a point source onto a perfectly rough, rigid support as shown in Fig. 5. We assume that this construction leads to a series of shallow surface avalanches whereby all grains have come to rest, at the point of burial, very close to the free surface of the pile. (Very different conditions may apply for wedges of sand; see Ref. [15].) An alternative history is the sieved pile, which is a cone created by sieving a series of concentric 
discs one on top of the other. In the standard sandpile, it is well known that the vertical normal stress has a minimum, not a maximum, beneath the apex. 32,33 A striking feature of our modelling approach is that it not only accounts for this "stress-dip" reasonably well, but predicts that it should be entirely absent for a sieved pile. This proposal is currently being subject to careful experimental verification. [34]

\section{A. Continuum Modelling of Granular Media}

The equations of stress continuity express the fact that, in static equilibrium, the forces acting on a small element of material must balance. For a conical pile of sand we have, in $d=3$ dimensions

$$
\begin{gathered}
\partial_{r} \sigma_{r r}+\partial_{z} \sigma_{z r}=\beta\left(\sigma_{\chi \chi}-\sigma_{r r}\right) / r \\
\partial_{r} \sigma_{r z}+\partial_{z} \sigma_{z z}=g-\beta \sigma_{r z} / r \\
\partial_{\chi} \sigma_{i j}=0
\end{gathered}
$$

where $\beta=1$. Here $z, r$ and $\chi$ are cylindrical polar coordinates, with $z$ the downward vertical. We take $r=0$ as a symmetry axis, so that $\sigma_{r \chi}=\sigma_{z \chi}=0 ; g$ is the force of gravity per unit volume; $\sigma_{i j}$ is the usual stress tensor which is symmetric in $i, j$. The equations for $d=2$ are obtained by setting $\beta=0$ in (3, 团) and suppressing (5). These describe a wedge of constant cross section and infinite extent in the third dimension.

The Coulomb law states that, at any point in a cohesionless granular medium, the shear force acting across any plane must be smaller in magnitude than $\tan \phi$ times the compressive normal force. Here $\phi$ is the angle of friction, a material parameter which, in simple models, is equal to the angle of repose. We accept this here, while noting that (i) $\phi$ in principle depends

on the texture (or fabric) of the medium and hence on its construction history; (ii) for a highly anisotropic packing, the existence of an orientation-independent $\phi$ is questionable; (iii) the identification of $\phi$ with the repose angle ignores some complications such as the 
Bagnold hysteresis effect (which may in turn be coupled to density changes). Setting these to one side, we note that the Coulomb law is an inequality: therefore, when combined with stress continuity, it cannot lead to closed equations for the granular stresses. To close the system of equations, further assumptions are clearly required. One choice is to assume that the material is an elastic continuum wherever it does not violate the Coulomb condition. (This is the simplest possible type of elastoplastic model.) A second choice it to treat the Coulomb condition as though it were an equality. This is the basis of the so-called "rigid plastic" approach to granular media. We return to both of these modelling schemes after first describing our own approach.

\section{Constitutive Relations Among Stresses}

We view cohesionless granular matter as assembly of rigid particles held up by friction. The static indeterminacy of frictional forces can, we argue, then be circumvented by assuming the existence of some local constitutive relations (c.r.'s) among components of the stress tensor. [7 9] The c.r.'s among stress components are taken to encode the network of contacts in the granular packing geometry; they therefore depend explicitly on its construction history. The task is then to postulate and/or justify physically suitable c.r.'s among stresses, of which only one (the primary c.r.) is required for systems with two dimensional symmetry, such as a wedge of sand; for a three dimensional symmetric assembly (the conical sandpile) a secondary c.r. is also needed.

The above nomenclature has caused confusion to some commentators on our work. In solid mechanics the term 'constitutive relation' normally refers to a material-dependent equation relating stress and strain. In fluid mechanics one has instead equations relating stress and (in the general case of a viscoelastic fluid) strain-rate history. Instead, our models of granular media entail equations relating stress components to one another, in a manner that we take to depend on the construction history of the material. Clearly such equations are intended to describe constitutive properties of the medium: they relate its state of stress to 
other discernable features of its physical state. We see no alternative to the term 'constitutive relations' for such equations.

In the simplest case, which is the FPA model [8,9] one hypothesizes that, in each material element, the orientation of the stress ellipsoid became 'locked' into the material texture at the time when it last came to rest, and does not change in subsequent compatible loadings. This is a bold, simplifying assumption, and it may indeed be far too simple, but it exemplifies the idea of having a local rule for stress propagation that depends explicitly on construction history. At first sight the idea of 'locking in' the principal axes seems to contradict the conception of an adaptive granular skeleton which can rearrange in response to small incremental loads. Remember though that this 'locking in' only applies for compatible loads - those which the existing skeleton can support. Any incompatible load will cause reorganization. We therefore require that any incompatible loads are specified in defining the construction history of the material.

For the standard sandpile geometry (see Fig. 5), where the material comes to rest on a surface slip plane, such loads do not in fact arise after material is buried. The FPA constitutive hypothesis then leads to the following primary c.r. among stresses:

$$
\sigma_{r r}=\sigma_{z z}-2 \tan \phi \sigma_{z r}
$$

where $\phi$ is the angle of repose. Eq.(6) is algebraically specific to the case of a standard sandpile created from a point source by a series of avalanches along the free surface. The conceptual basis of the FPA model is not so narrow: indeed, we applied it already to jammed colloids in Section [1. More generally the FPA model is arguably the simplest possible choice for a history-dependent c.r. among stresses; but this does not mean it will be sensible in all geometries.

A consequence of Eq. (6) for a standard sandpile, is that the major principal axis everywhere bisects the angle between the vertical and the free surface. It should be noted that in cartesian coordinates, the FPA model reads:

$$
\sigma_{x x}=\sigma_{z z}-2 \operatorname{sign}(x) \tan \phi \sigma_{x z}
$$


where $x= \pm r$ is horizontal. From Eq. (7), the FPA constitutive relation is seen to be discontinuous on the central axis of the pile: the local texture of the packing has a singularity on the central axis which is reflected in the stress propagation rules of the model. (This is physically admissible since the centreline separates material which has avalanched to the left from material which has avalanched to the right.) The paradoxical requirement, on the centreline, that the principal axes are fixed simultaneously in two different directions

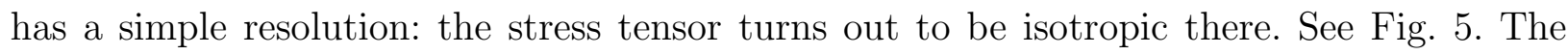
constitutive singularity leads to an 'arching' effect for the standard sandpile, as previously put forward by Edwards and Oakeshott [19] and others. [11,35]

The FPA model is one of a larger class of OSL (for "oriented stress linearity") models in which the primary constitutive relation (in the sandpile geometry) is, in Cartesians

$$
\sigma_{x x}=\eta \sigma_{z z}+\mu \operatorname{sign}(x) \sigma_{x z}
$$

with $\eta, \mu$ constants. Note that the boundary condition, that the free surface of a pile at its angle of repose $\phi$ is a slip plane, yields one equation linking $\eta$ and $\mu$ to $\phi$; thus, for a sandpile geometry, the OSL scheme represents a one-parameter family of primary c.r.'s. The OSL models were developed [9] to explain experimental data on the stress distribution beneath a standard sandpile. [32,33,36] With a plausible choice of secondary c.r. (of which several were tried, with only minor differences resulting), the experimental data (Fig. 6) is found to support models in the osL family with $\eta$ close, but perhaps not exactly equal, to unity (the FPA value). This is remarkable, in view of the radical simplicity of the assumptions made.

As explained by Wittmer et al., [8,9] the osL models, combined with stress continuity (Eq. 8) yield hyperbolic equations having fixed characteristic rays for stress propagation. In fact they are wave equations; [7.9] moreover they are essentially equivalent to Eq. (2), with (in general) an oblique triplet of directors (these become mutually orthogonal only in the case of FPA). The constitutive property that OSL models describe is that these characteristic rays (and not, in general, the principal axes) have orientations that are 'locked in' on burial 
of an element, and do not change when a further compatible load is applied. As demonstrated already in Section [1], there is every reason to identify such characteristics, in the continuum model, with the mean orientations of force chains in the underlying material.

Note that unless the OSL parameter is chosen so that $\mu=0$, a constitutive singularity on the central axis, as mentioned above for the FPA case, remains. (The characteristics are asymmetrically disposed about the vertical axis, and invert discontinuously at the centreline $x=0$.) The case $\mu=0$ corresponds to one studied earlier by Bouchaud et al., [7] and of the OSL family it is the only candidate for describing a sieved pile, in which the construction history clearly cannot lead to a constitutive singularity at the axis of the pile. The resulting 'BCC' model could be called a 'local Janssen model' in that it assumes local proportionality of horizontal and vertical compressive stresses - an assumption which, when applied globally to average stresses in a silo, was first made by Janssen. [37] The BCC model predicts a smooth maximum, not a dip, in the pressure beneath the apex of a pile. This is what we expect, therefore, in the case of a sieved pile. 9]

\section{Rigid-Plastic Models}

A more traditional, but related, approach is one based on the (Mohr-Coulomb) rigidplastic model. [38] To find so-called limit state solutions in this model, one postulates that the Coulomb condition is everywhere obeyed with equality. [39] That is, through every point in the material there passes some plane across which the shear force is exactly $\tan \phi$ times the normal force. By assuming this, the model allows closure (modulo a sign ambiguity discussed below) of the equations for the stress without invocation of an elastic strain field.

This limit-state analysis of the rigid plastic model is equivalent to assuming a 'constitutive relation' (sometimes called 'incipient failure everywhere' [9]):

$$
\sigma_{r r}=\sigma_{z z} \frac{1}{\cos ^{2} \phi}\left[\sin ^{2} \phi+1 \pm 2 \sin \phi \sqrt{1-\left(\cot \phi \sigma_{z r} / \sigma_{z z}\right)^{2}}\right]
$$

whereas the Coulomb inequality requires only that $\sigma_{r r}$ lies between the two values $( \pm)$ on the right. It is a simple exercise to show that for a sandpile at its repose angle, only one 
solution of the resulting equations exists in which the sign choice is everywhere the same. This requires the negative root (conventionally referred to as an 'active' solution) and it shows a hump, not a dip, in the vertical normal stress beneath the apex. Savage [10], however, draws attention to a 'passive' solution, having a pronounced dip beneath the apex. [39] The passive solution actually contains a pair of matching planes between an inner region where the positive root of (9) is taken, and an outer region where the negative is chosen. In fact (see Ref. [15]) there are more than one such matched solutions, corresponding to different types of discontinuity in the stress (or its gradient) at the matching plane and/or the pile centre. Moreover, there is no physical principle that limits the number of matching surfaces; by adding extra ones, a very wide variety of results might be achieved.

It is interesting to compare the mathematics, and physics, of Eq. (9) with that of the OSL models introduced above. The rigid-plastic model yields a local c.r. among stresses; like OSL the resulting equations are hyperbolic. It also exhibits fragility: because a yield plane passes through every material point, certain incremental loads will cause reorganization. Therefore, anyone who defends the rigid-plastic model as a cogent description of sandpiles cannot reasonably object to these same features in our own models. Conversely, we cannot object in principle to a model in which a Coulomb yield plane passes through every material point. However, we still see no reason why it should be a good model; [9] in particular we cannot see how to make a link between the characteristic rays in this model (which are always load dependent) and the underlying geometry of the contact network in the medium. In contrast, this link arises naturally in the OsL framework.

\section{Elastoplastic models}

The simplest elastoplastic models assume a material in which a perfectly elastic behaviour at small strains is conjoined onto perfect plasticity (the Coulomb condition with equality) at larger ones. In such an approach to the standard sandpile, an inner elastic region connects

onto an outer plastic one. In the inner elastic region the stresses obey the Navier equations, 
which follow from those of Hookean elasticity by elimination of the strain variables. The corresponding strain field is usually not discussed, but tacitly treated as infinitesimal: the high modulus limit is taken. It has been argued that, for a sandpile on a rigid support FPAlike solutions can be found within a purely elastoplastic model, at least in two dimensions. 40] However, since these show a cusp in the vertical stress on the centreline, they imply an infinitesimal displacement field incompatible with a continuous elasticity across the midline.

[3] On the other hand, it is possible to obtain a stress dip, in an elastoplastic model, by assuming that the supporting base is not rigid but subject to basal sag. [3] This explanation cannot explain the data of Huntley [33] which involves an indentable (rather than sagging) base. 15 Moreover, it would predict a similar dip for a sieved pile, unlike or own models; experiments on this point are now available, and suggest that indeed no dip is seen in this case. 34

Objections to the elastoplastic approach to modelling sandpiles can also be raised at a much more fundamental level. [16,15] Specifically, to make unambiguous predictions for the stresses in a sandpile, these models require boundary information which, at least for the simpler models, can be given no clear physical meaning or interpretation. We return to this point below.

\section{B. Fragile vs. Elastoplastic Descriptions}

\section{Problems definining an elastic strain}

In the FPA model and its relatives, strain variables are not considered. No elastic modulus enters, and there is no intrinsic stress scale. The resulting predictions for a conical pile therefore obey what is usually called radial stress-field (RSF) scaling. Formally one has for the stresses at the base

$$
\sigma_{i j}=g h s_{i j}(r / c h)
$$


where $h$ is the pile height, $c=\cot \alpha$ and $s_{i j}$ a reduced stress: $\alpha$ is the angle between the free surface and the horizontal so that for a pile at repose, $\alpha=\phi$. This form of RSF scaling, which involves only the forces at the base, [1] might be called the 'weak form' and is distinct from the 'strong form' in which Eq. (10) is obeyed also with $z$ (an arbitrary height from the apex) replacing $h$ (the overall height of the pile). Our OsL models obey both forms; only the weak form has been tested directly by experiment but it is well-confirmed in many systems (Smid and Novosad [32], Huntley [33]).

The observation of RSF scaling, to experimental accuracy, suggests that elastic effects need not be considered explicitly. This does not of itself rule out elastic or elastoplastic behaviour which, at least in the limit of large modulus, can also yield equations for the stress from which the bulk strain fields, and hence also the modulus, cancel. (Note that it is tempting, but entirely wrong, to assume that a similar cancellation occurs at the boundaries of the material; we return to this below.) The cancellation of bulk strain fields in elastoplastic models disguises a serious problem in their application to the standard sandpile and related geometries. [15,16] The difficulty is this: there is no obvious definition of strain or displacement for such a construction history. To define a physical displacement or strain field, one requires a reference state. In (say) a triaxial strain test (see, e.g., Ref. [42]) an initial state is made by some reproducible procedure, and strains measured from there. The elastic part is identifiable in principle, by removing the applied stresses (maintaining an isotropic pressure) and seeing how much the sample recovers its shape. In contrast, a pile constructed by pouring grains onto its apex is not made by a plastic and/or elastic deformation from some initial reference state of the same continuous medium. The problem of the missing reference state occurs whenever the solidity of the body itself arises purely because of the load applied. Thus, for the jammed colloid considered in Section $\mathbb{\text { II }}$ above, the unloaded state is simply a fluid. For the sandpile, it is grains floating freely in space. On cannot satisfactorily define an elastic strain with respect to either of these reference states.

A route to defining a strain variable does however exist, [2] so long as one ignores the fact that tensile forces are prohibited. In effect, one assumes that when grains of sand arrive 
at the free surface, each one forms permanent or "glued" elastic contacts with its neighbours; [16] this contact network can then, by assumption, elastically support arbitrary incremental loads. This is an admissable physical hypothesis, though contradictory to our own hypothesis of an adaptive, fragile granular skeleton. We do not yet know which hypothesis is more correct; the test of this lies in experiment. (It does not lie in a sociological comparison of how physicists and engineers approach their work, as offered by Savage. [3])

If the "glued pile" model is correct, then a strain variable is defined from the relative displacement that has occurred between adjoining particles since the moment they first were glued together. [2] However, the resulting displacement field, found by integrating the strain, is unlikely to be single-valued. Put differently, if a glued assembly is created under gravity and then gravity is switched off, it will revert to a state in which there are residual elastic strains throughout the material, even though there is now no body force acting (Fig. 9(a)). This is because the particle contact network was itself created under partially-loaded conditions. Many elastic and elastoplastic calculations, such as all those reviewed by Savage, [3] entirely ignore the problem of quenched stresses, and therefore embody an implausible "floating model" of a sandpile shown in Fig. 9 (b).

Note that these effects do not become small when the limit of a large modulus is taken; the quenched stresses remain of order the stress that was acting during formation, and can take both signs (tensile as well as compressive). [23] So, if one creates a glued pile under gravity and then slowly switches off the body force, tensile forces will arise long before $g$ has gone to zero. In this sense, the response to gravity of a cohesionless pile is completely nonlinear. Correspondingly, in an unglued pile, no smooth deformation can connect the state of a pile created under gravity with an unloaded state of the same contact network: as the load is removed, such a pile will undergo large-scale reorganization. 


\section{Boundary conditions and determinacy in hyperbolic models}

Models, such as OSL, that assume local constitutive equations among stresses provide hyperbolic differential equations for the stress field. Accordingly, if one specifies a zero-force boundary condition at the free (upper) surface of a granular aggregate on a rough rigid support, then any perturbation arising from a small extra body force (in two dimensions, for simplicity) propagates along two characteristics passing through this point. In the OSL models these characteristics are, moreover, straight lines. Therefore the force at the base can be found simply by summing contributions from all the body forces as propagated along two characteristic rays onto the support; the sandpile problem is, within the modelling approach by Bouchaud et al. [7] and Wittmer et al., [8,9] mathematically well-posed. There is no need to consider any elastic strain field and the paradoxes concerning its definition in cohesionless poured sand, discussed above, do not arise.

Note that in principle, one could have propagation also along the 'backward' characteristics (see Fig. $7(\mathrm{a})$ ). This is forbidden since these cut the free surface; any such propagation can only arise in the presence of a nonzero surface force, in violation of the boundary conditions. Therefore the fact that the propagation occurs only along downward characteristics is not related to the fact that gravity acts downward; it arises because we know already the forces acting at the free surface (they are zero). Suppose we had instead an inverse problem: a pile on a bed with some unspecified overload at the top surface, for which the forces acting at the base had been measured. In this case, the information from the known forces could be propagated along the upward characteristics to find the unknown overload. More generally, in OSL models, each characteristic ray will cut the surface of a (convex) patch of material at two points; the sum of the forces along the ray at the two ends must then be balanced by the longitudinal component of the body force integrated along the ray (see Fig. 7 (b)). These models are thus "boundary fragile".

In three dimensions, the mathematical structure of these models is somewhat altered, [7] but the conclusions are basically unaffected. The propagation of stresses is governed by a 
Green's function which is the response to a localized overload; OsL models predict that for (say) sand in a horizontal bed, the maximum response at the base is not directly beneath a localized overload but on a ring of finite radius (proportional to the depth) with this as its axis. [7] (This could be difficult to test cleanly because of noise effects, but there are related consequences for stress-stress correlations which are discussed in Ref. [13, 14].) On the other hand, for different geometries, such as sand in a bin, the stress propagation problem is not well-posed even with hyperbolic equations, unless something is known about the interaction between the medium and the sidewalls. But by assuming a constant ratio to shear and normal forces at the walls, further interesting predictions can be made, for example that the total weight increment measured at the base of a cylindrical silo, in response to an overload on the top, is a nonmonotonic function of the height of the fill. 43] These predictions represent clear signatures of hyperbolic stress propagation and, if confirmed experimentally, would be hard to explain by other means.

\section{The problem of elastic indeterminacy}

The well-posedness of the standard sandpile is not shared be models involving the elliptic equations for an elastic body. For such a material, the stresses throughout the body can be solved only if, at all points on the boundary, either the force distribution or a displacement field is specified. 44] Accordingly, once the zero-stress boundary condition is applied at the free surface, nothing can in principle be calculated unless either the forces or the displace-

ments at the base are known (and the former amounts to specifying in advance the solution of the problem). The problem does not arise from any uncertainty about what to do mathematically: one should specify a displacement field at the base. Difficulties nonetheless arise if, as we argued above, no physical significance can be attributed to this displacement field for cohesionless poured sand.

To give a specific example, consider the static equilibrium of an elastic cone of finite modulus, which is placed in an unstressed state (without gravity) onto a completely rough, 
rigid surface; gravity is then switched on. This generates a pressure distribution with a smooth parabolic hump as in Fig. Ba. (The roughness can crudely be represented by a set of pins.) Starting from any initial configuration, another can be generated by pulling and pushing parts of the body horizontally across the base (i.e., changing the displacements there); if this is rough, the new state will still be pinned and will achieve a new static equilibrium. This will generate a stress distribution, across the supporting surface and within the pile, that differs from the original one. Indeed, if a large enough modulus is now taken (at fixed forces), this procedure allows one to generate arbitrary differences in the stress distribution while generating neither appreciable distortions in the shape of the cone, nor any forces at its free surface. This corresponds to a limit $Y \rightarrow \infty, \underline{u} \rightarrow 0$ at fixed $Y \underline{u}$ where $Y$ is the modulus and $\underline{u}$ the displacement field at the base.

Analogous remarks apply to any simple elastoplastic theory of sandpiles, in which an elastic zone, in contact with part of the base, is attached at matching surfaces to a plastic zone. A natural presumption for the standard sandpile might be that $Y \underline{u}=0$ (that is, the basal displacements vanish before the high modulus limit is taken). This is consistent with the "glued pile" interpretation of elastic models - one assumes that glue also firmly attaches grains to the support as they arrive. However, the same interpretation, as shown above, also requires explicit consideration of quenched stresses (see Fig. 9). Note in any case that elastic and elastoplastic predictions for the sandpile are indeterminate, in a rigorous mathematical sense, if the $Y \rightarrow \infty$ limit is taken before the basal displacements $\underline{u}$ have been specified.

Experiments (reviewed in detail in Cates et al. [15]) report that for sandpiles on a rough rigid support, the forces on the base can be measured reproducibly; and, although subject to statistical fluctuations on the scale of several grains, do not vary too much among piles constructed in the same way. In contrast, for any simple elastic or elastoplastic model that does not include a specification of the basal displacements, there is a very large indeterminacy in the predicted stress distribution, even after averaging over any statistical fluctuations. An elastoplastic modeller who believes that the experiments measure something well-defined is then obliged to explain why and how the basal displacements (even if infinitesimal) are fixed 
by the construction history. Note that basal sag [3,10] is not a candidate for the missing mechanism, since it does not resolve the elastic indeterminacy in these models; the latter arises primarily from the roughness, rather than the rigidity, of the support. An alternative view is that of Evesque, [45] who directly confronts the issue of elastic indeterminacy and seemingly concludes that the experimental results themselves are and must be indeterminate; he argues that the external forces acting on the base of a pile are effectively chosen at will, rather than actually measured, by the experimentalist (see also Ref. 46]). To what extent this viewpoint is based on experiment, and to what extent on an implicit presumption in favour of elastoplastic theory, is to us unclear.

\section{Crossover from Fragile to Elastic Regimes}

We have emphasized above the very different modelling assumptions of the fragile and elast(oplast)ic approaches to granular media. However, we have recently shown that hyperbolic fragile behaviour can be recovered from an elastoplastic description by taking a strongly anisotropic limit. [16] Moreover, the crossover between elastic and hyperbolic behaviour, at least for one simple model of the granular skeleton, [16] is controlled by the deformability of the granular particles. For simplicity in this section, we restrict attention to the FPA model.

The FPA model describes, by definition, a material in which the shear stress must vanish across a pair of orthogonal planes fixed in the medium - those normal to the (fixed) principal axes of the stress tensor. According to the Coulomb inequality (which we also assume) the shear stress must also be less than $\tan \phi$ times the normal stress, across planes oriented in all other directions. Clearly this combination of requirements can be viewed as a limiting case of an elastoplastic model with an anisotropic yield condition:

$$
\left|\sigma_{t n}\right| \leq \sigma_{n n} \tan \Phi(\theta)
$$

where $\theta$ is the angle between the plane normal $\mathbf{n}$ and the vertical (say) and $\mathbf{t} \cdot \mathbf{n}=0$. An anisotropic yield condition should arise, in principle, in any material having a nontrivial 
fabric, arising from its construction history. The limiting choice corresponding to the FPA model for a sandpile is $\Phi(\theta)=0$ for $\theta=(\pi-2 \phi) / 4$ (this corresponds to planes where $\mathbf{n}$ lies parallel to the major principal axis), and $\Phi(\theta)=\phi$ otherwise. (There is no separate need to specify the second, orthogonal plane across which shear stresses vanish, since this is assured by the symmetry of the stress tensor.) By a similar argument, all other osL models can also be cast in terms of an anisotropic yield condition, of the form $\left|\sigma_{t n}-\sigma_{n n} \tan \Psi(\theta)\right| \leq \sigma_{n n} \tan \Phi(\theta)$ where $\Phi(\theta)$ vanishes, and $\Phi(\theta)$ is finite for two values of $\theta$. (This fixes a nonzero ratio of shear and normal stresses across certain special planes.)

At this purely phenomenological level there is no difficulty in connecting hyperbolic models smoothly onto highly anisotropic elastoplastic descriptions. Specifically, consider a medium having an orientation-dependent friction angle $\Phi(\theta)$ that does not actually vanish, but is instead very small ( $\leq \epsilon$, say) in a narrow range of angles (say of order $\epsilon$ ) around $\theta=(\pi-2 \phi) / 4$, and approaches $\phi$ elsewhere. (One interesting way to achieve the required yield anisotropy is to have a strong anisotropy in the elastic response, and then impose a uniform yield condition to the strains, rather than stresses.)

Such a material will have, in principle, mixed elliptic/hyperbolic equations of the usual elastoplastic type. The resulting elastic and plastic regions must nonetheless arrange themselves so as to obey the FPA model to within terms that vanish as $\epsilon \rightarrow 0$. If $\epsilon$ is small but finite, then for this elastoplastic model the results will depend on the basal boundary condition, but only through these higher order corrections to the leading (FPA) result. Thus, although elastoplastic models do suffer from elastic indeterminacy (they require a basal displacement field to be specified), the extent of the influence of the boundary condition on the solution depends on the model chosen. Strong enough (fabric-dependent) anisotropy, in an elastoplastic description, might so constrain the solution that it is primarily the granular fabric (hence the construction history) and only minimally the boundary conditions which actually determine the stresses in the body. For models such as that given above there is a well-defined limit where the indeterminacy is entirely lifted, hyperbolic equations are recovered, and it is quite proper to talk of local stress propagation 'rules' determined by the 
construction history of the material. Our continuum modelling framework is based precisely on these assumptions.

The crossover just outlined can also be understood directly in terms of the micromechanics of force chains, at least within the simplified picture developed in Section [1]. We consider a regular lattice of force chains (see Fig. 2 (b)), for simplicity rectangular (the FPA case), which is fragile if the chains can support only longitudinal forces. As mentioned in Section 【IIC, this is true so long as such paths consist of linear chains of rigid particles, meeting at frictional point contacts: the forces on all particles within each chain must then be colinear, to avoid torques. This imposes the (FPA) requirement that there are no shear forces across a pair of orthogonal planes normal to the force chains themselves. Suppose now a small degree of particle deformability is introduced. [16] This relaxes slightly the collinearity requirement, but only because the point contacts are now flattened (see Fig. 3 (b)). The ratio $\epsilon$ of the maximum transverse load to the normal one will therefore vanish with (some power of) the mean compression. This yield criterion applies only across two special planes; failure across others is governed by some smooth yield requirement (such as the ordinary Coulomb condition: the ratio of the principal stresses lies between given limits). The granular skeleton just described, which was fragile in the limit of rigid grains, is now governed by a strongly anisotropic elastoplastic yield criterion of precisely the kind described above. This indicates how a packing of frictional, deformable rough particles, displaying broadly conventional elastoplastic features when the deformability is significant, can approach a fragile limit when the limit of a large modulus is taken. (It does not prove that all packings become fragile in this limit.) Conversely it shows how a packing that is basically fragile in its response to a graviational load could nonetheless support very small incremental deformations, such as sound waves, by an elastic mechanism.

The question of whether sandpiles are better described as fragile, or as ordinarily elastoplastic, remains open experimentally. To some extent it may depend on the question being asked. However, we have argued, on various grounds, that in calculating the stresses in a pile under gravity a fragile description may lie closer to the true physics. 


\section{CONCLUSIONS}

The jammed state of colloids, if it indeed exists in the laboratory, has not yet been fully elucidated by experiment. It is interesting that even very simple models, such as Eq. (11), can lead to nontrivial and testable predictions (such as the constancy of certain measured stress ratios). Such models suggest an appealing conceptual link between jamming, force chains, and fragile matter. 15,16] However, further experiments are needed to establish the degree to which they are useful in describing real colloids.

For granular media, the existence of tenuous force-chain skeletons is clear; [曰 6, 17,24] the question is whether such skeletons are fragile. Several theoretical arguments have been given, above and elsewhere, to suggest that this may be the case, at least in the limit of rigid particles. Moreover, simulations show strong rearrangement under small changes of compression axis; the skeleton is indeed "self-organized". [5,6] Experiments also suggest cascades of rearrangement 27,31] in response to small disturbances. These findings are consistent with the fragile picture.

The standard sandpile (a conical pile formed by pouring onto a rough rigid support) has played a central role in our discussions. From the perspective of geotechnical engineering, the problem of calculating stresses in the humble sandpile may appear to be of only of marginal importance. The physicist's view is different: the sandpile is important, because it is one of the simplest problems in granular mechanics imaginable. It therefore provides a test-bed for existing models and, if these show shortcomings, may suggest ideas for improved physical theories of granular media.

Given the present state of the data, a conventional elastoplastic interpretation of the experimental results for sandpiles may remain tenable; more experiments are urgently required. [34] In the mean time, a desire to keep using tried-and-tested modelling strategies until these are demonstrably proven ineffective is quite understandable. We find it harder to accept the suggestion [10] that anyone who questions the general validity of traditional elastoplastic thinking is somehow uneducated. 
In summary, we have discussed a new class of models for stress propagation in granular matter. These models assume local propagation rules for stresses which depend on the construction history of the material and which lead to hyperbolic differential equations for the stresses. As such, their physical basis is substantially different from that of conventional elastoplastic theory. Our approach predicts 'fragile' behaviour, in which stresses are supported by a granular skeleton of force chains that respond by finite internal rearrangement to certain types of infinitesimal load. Obviously, such models of granular matter might be incomplete in various ways. Specifically we have discussed a possible crossover to elastic behaviour at very small incremental loads, and to conventional elastoplasticity at very high mean stresses (when significant particle deformations arise). However, we believe that our approach, by capturing at the continuum level at least some of the physics of force chains, may offer important insights that lie beyond the scope of previous continuum modelling strategies.

\section{ACKNOWLEDGMENT}

We thank R. C. Ball, E. Clement, C. S. and M. J. Cowperthwaite, S. F. Edwards, P. Evesque, P.-G. de Gennes, G. Gudehus, J. Goddard, D. Levine, C. E. Lester, J. Melrose, S. Nagel, F. Radjai, J.-N. Roux, J. Socolar, C. Thornton, L. Vanel and T. A. Witten for illuminating discussions. Work funded in part by EPSRC (UK) GR/K56223 and GR/K76733. 


\section{REFERENCES}

[1] E. Guyon, S. Roux, A. Hansen, D. Bideau, J.-P. Troadec, and H. Crapo, Reports on Progess in Physics, 53, 373 (1990).

[2] For recent discussions see P.-G. de Gennes, Physica A, 261, 267 (1998); P. Evesque and P.-G. de Gennes, Compt. Rend. de l'Acad. des Sci., 326, 761 (1998).

[3] S. B. Savage, in Proc. Physics of dry granular media, Cargese, France, October 1997 edited by H.J. Herrmann, J.P. Hovi and S. Luding (NATO Advanced Study Institute, Kluwer, 1998), pp. 25-96.

[4] P. Dantu, Annales des Ponts et Chaussées, 4, 144 (1967).

[5] C. Thornton, in KONA Powder and Particle 15 (1997); C. Thornton and G. Sun, in Numerical Methods in Geotechnical Engineering edited by I. Smith (Balkema, Rotterdam, 1994), pp. 143-148.

[6] F. Radjai, D. E. Wolf, M. Jean, J. J. Moreau, Phys. Rev. Lett. 90, 61 (1998); F. Radjai, S. Roux, J. J. Moreau, within this volume.

[7] J.-P. Bouchaud, M. E. Cates, and P. Claudin, J. Physique I, 5639 (1995).

[8] J. P. Wittmer, P. Claudin, M. E. Cates and J.-P. Bouchaud, Nature, 382, 336 (1996).

[9] J. P. Wittmer, P. Claudin, M. E. Cates, J. Physique I, 7, 39 (1997).

[10] S. B. Savage, in Proc. 3rd Int. Conf. on Powders and Grains, Durham NC 18-23 May 1997, edited by R. P. Behringer \& J. T. Jenkins (Balkema, Rotterdam, 1997), pp. 185194.

[11] D.H. Trollope, in Rock Mechanics in Engineering Practice edited by K. G. Stagg and O. C. Zienkiewicz (Wiley, New York, 1968), ch. 9, pp. 275-320.

[12] M. E. Harr, Mechanics of Particulate Media (McGraw Hill, New York, 1977). 
[13] J.-P. Bouchaud, P. Claudin, M. E. Cates, J. P. Wittmer, in Physics of Dry Granular Media, edited by H.J. Herrmann, J.P. Hovi and S. Luding (NATO Advanced Study Institute, Kluwer, 1997), pp. 97-122.

[14] P. Claudin, J.-P. Bouchaud, M. E. Cates and J. P. Wittmer, Phys. Rev. E, 57, 4441 (1998).

[15] M. E. Cates, J. P. Wittmer, J.-P. Bouchaud, P. Claudin, Phil. Trans. Roy. Soc. Lond. Ser. A., 356, 2535 (1998).

[16] M. E. Cates, J. P. Wittmer, J.-P. Bouchaud, P. Claudin, Phys. Rev. Lett. 81, 1841 (1998).

[17] D. Kolymbas, Archive of Applied Mechanics., 61, 143 (1991); D. Kolymbas and W. Wu, in Modern Approaches to Plasticity edited by D. Kolymbas (Elsevier, Rotterdam, 1985), pp. 213-223.

[18] G. Gudehus, in Proc. 3rd Int. Conf. on Powders and Grains, Durham NC, USA 18-23 May 1997 edited by R. P. Behringer \& J. T. Jenkins (Balkema, Rotterdam, 1997), pp. 169-183.

[19] S. F. Edwards and R. B. S. Oakeshott, Physica D 38, 88 (1989); S. F. Edwards and C. C. Mounfield, Physica A 226, 1, 12, 25 (1996); S. F. Edwards, Physica A 249, 226 (1998).

[20] R. C. Ball and S. F. Edwards, in preparation; D. V. Grinev and R. C. Ball, in preparation.

[21] S. Alexander, Phys. Reports 296, 65 (1998); P. S. Cundall and O. D. L. Strack, in Mechanics of Granular Materials: New Models and Constitutive Relations edited by J. T. Jenkins and M. Satake (Elsevier, Rotterdam, 1983).

[22] P. Bak, How Nature Works; The Science of Self-Organized Criticality (Oxford University 
Press, Oxford 1997).

[23] C. Moukarzel, PRL, 81, 1634 (1998); to appear in Proc. of Rigidity theory and applications, Traverse City MI, June 14-18 1998, Fundamental Material Science Series, Plenum (cond-mat/9807004).

[24] C. H. Liu, S. R. Nagel, D. A. Schecter, S. N. Coppersmith, S. Majumdar and T. A. Witten, Science 269513 (1995).

[25] A. V. Thachenko, T. A. Witten, cond-mat/9811171.

[26] S. Ouaguenouni, J.N. Roux, Europhysics Letters, 39, 117 (1997).

[27] P. Claudin and J.-P. Bouchaud, Phys. Rev. Lett. 78, 231 (1997).

[28] H. M. Laun, J. Non-Newtonian Fluid Mech., 54, 87 (1994).

[29] R. S. Farr, J. R. Melrose, and R. C. Ball, Phys. Rev. E, 55, 7203 (1997); R. C. Ball, J. R. Melrose, Adv. Colloid Interface Sci., 59, 19 (1995); J. R. Melrose, R. C. Ball, Europhys. Lett., 32, 535 (1995). See also E. Aharonov, D. Sparks, cond-mat/9812204.

[30] R. Hooke, A description of helioscopes, and some other instruments John Martin, London (1676). See anagram on title page concerning "The true Mathematical and Mechanical form of all manner of Arches."; reproduced as Fig.2.2 of [42].

[31] E. Kolb, T. Mazozi, J. Duran, E. Clement, in preparation; P. Claudin, J.-P. Bouchaud, Granular Matter, 1, 71 (1998).

[32] J. Smid and J. Novosad, in Proc. of 1981 Powtech Conf., Ind. Chem. Eng. Symp., 63, D3V 1-12 (1981).

[33] R. Brockbank, J. M. Huntley, R. C. Ball, J. Physique II, 7, 1521 (1997).

[34] L. Vanel, D.W. Howell, E. Clément and R.P. Behringer, in preparation.

[35] D. H. Trollope and B .C. Burman, Geotechnique 30, 137 (1980). 
[36] T. Jokati, R. Moriyama, J. Soc. Powder Technol. Jpn. 60, 184 (1979).

[37] H. A. Janssen, Z. Vert. Dt. Ing. 39, 1045 (1895).

[38] R. M. Nedderman, Statics and Kinematics of Granular Materials Cambridge University Press 1992.

[39] Savage's claim that active and passive solutions can be 'generally regarded as bounds between which other states can exist, i.e., when the material is behaving in an elastic or elastoplastic manner' is disproved by counterexample in Ref. [15].

[40] F. Cantelaube and J. D. Goddard, in Proc. 3rd Int. Conf. on Powders and Grains, Durham NC, USA 18-23 May 1997 edited by R. P. Behringer \& J. T. Jenkins (Balkema, Rotterdam, 1997), pp. 231-234.

[41] P. Evesque, J. Physique I 7, 1305 (1997).

[42] D. Muir Wood, Soil Behaviour and Critical State Soil Mechanics (Cambridge University Press, Cambridge, 1990).

[43] P. Claudin, PhD Thesis, Université de Paris XI 1999.

[44] L. D. Landau, E. M. Lifshitz, Theory of Elasticity, 3rd Edn., Oxford: Pergamon.

[45] P. Evesque, private communication 1996.

[46] P. Evesque and S. Boufellouh, in Proc. 3rd Int. Conf. on Powders and Grains, Durham NC, USA 18-23 May 1997 edited by R. P. Behringer \& J. T. Jenkins (Balkema, Rotterdam, 1997), pp. 295-298. 


\section{FIGURES}
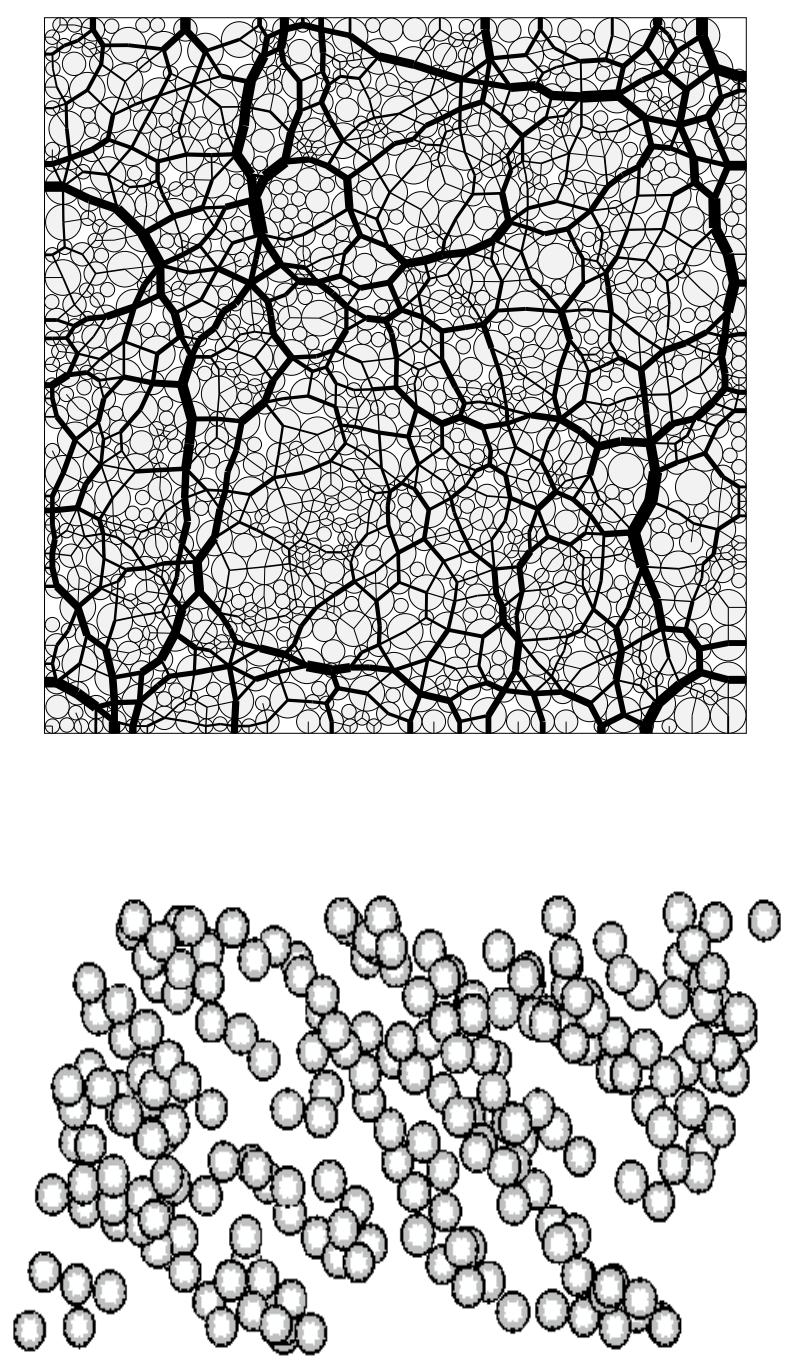

FIG. 1. (a) Granular skeleton in two-dimensional frictional hard spheres by F. Radjai et al. [6] (Figure courtesy of F. Radjai.) (b) The jamming transition in a sheared colloid. The data are from a computer simulation of a hard sphere colloidal suspension at $\phi=0.54$ which has been strained to $\gamma=0.22$. Shown in the figure are only those spheres which have come into very close contact $\left(\leq 10^{-5}\right.$ radius $)$ with at least one neighbour. As seen from the figure, the contact geometry is strongly anisotropic and suggests the formation of "force chains" running top left to bottom right. (The simulation is by J. Melrose, Cavendish Laboratory; the figure is courtesy of him.) 


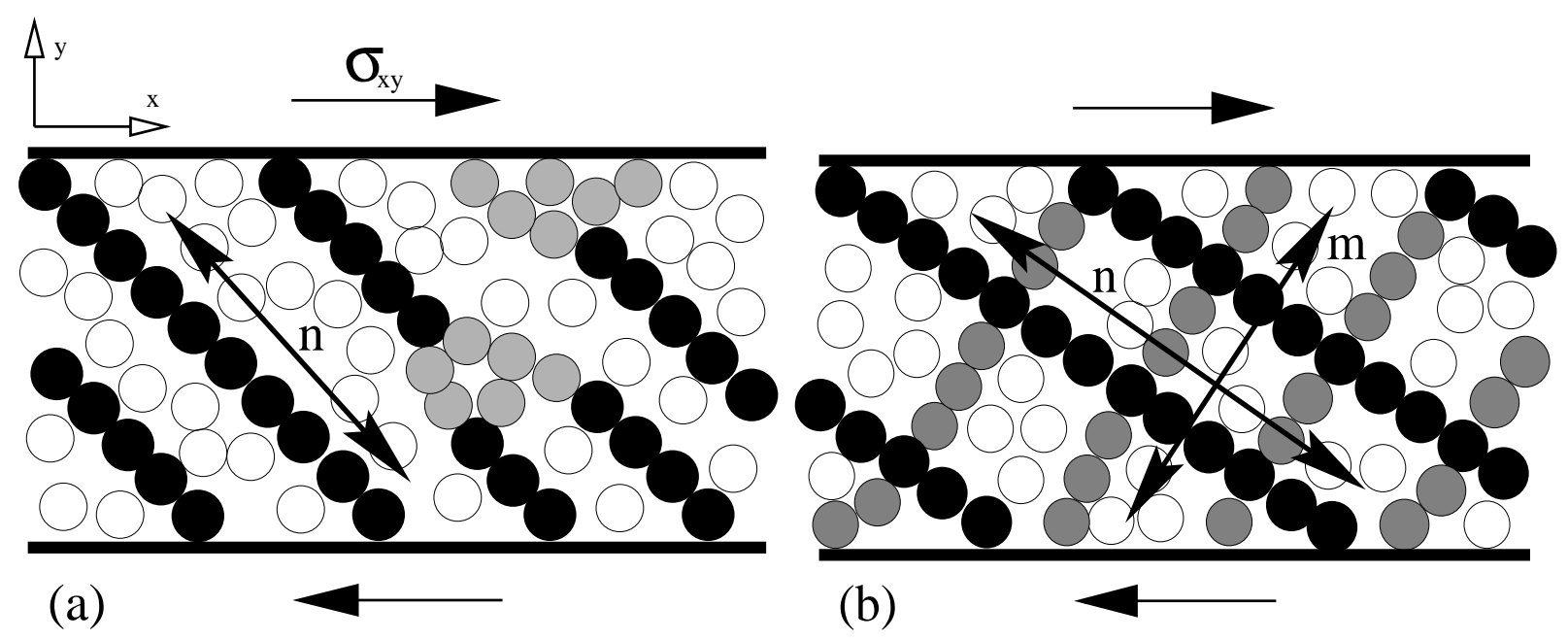

FIG. 2. (a) A jammed colloid (schematic). Black: force chains; grey: other force-bearing particles; white: spectators. (b) Idealized rectangular network of force chains. 


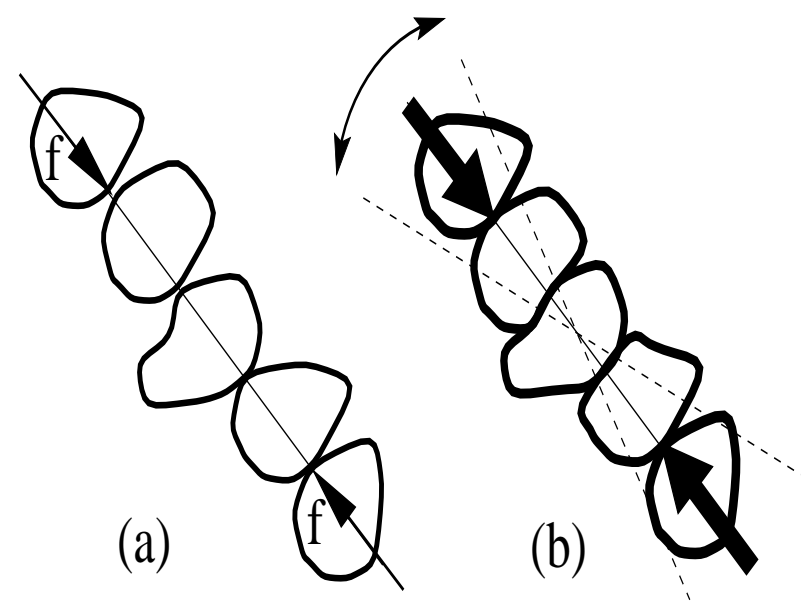

FIG. 3. (a) A force chain of hard particles (any shape) can statically support only longitudinal compression. Note that neither friction at the contacts, nor particle aspherity, can change this "longitudinal force" rule. (b) Finite deformability allows small transverse loads to arise. 


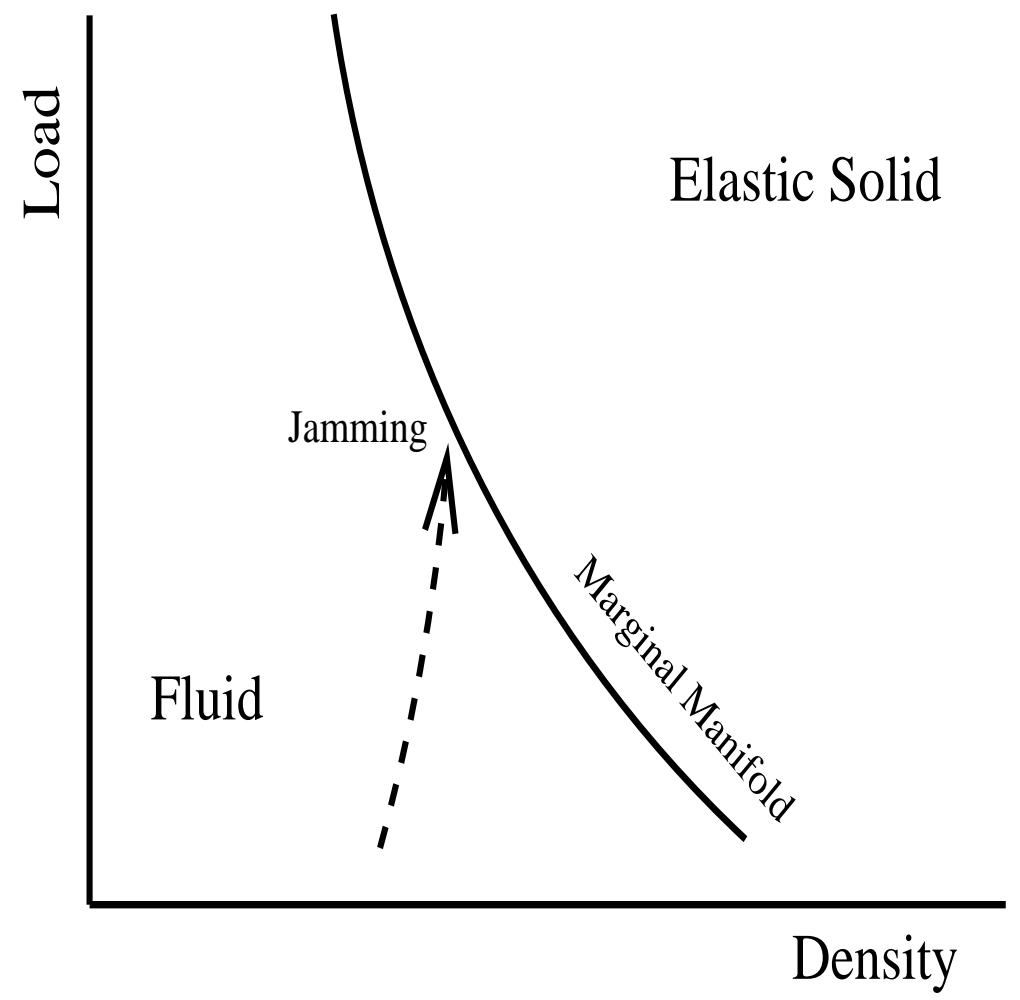

FIG. 4. Schematic "phase diagram" of a jamming system. If, as load or density is increased (dashed arrow), the granular skeleton arrests on first being able to support the applied load, it can remain indefinitely on the "marginal manifold" separating conventional solids from liquidlike packings. Incompatible loads will move the system to another point on the same manifold. 


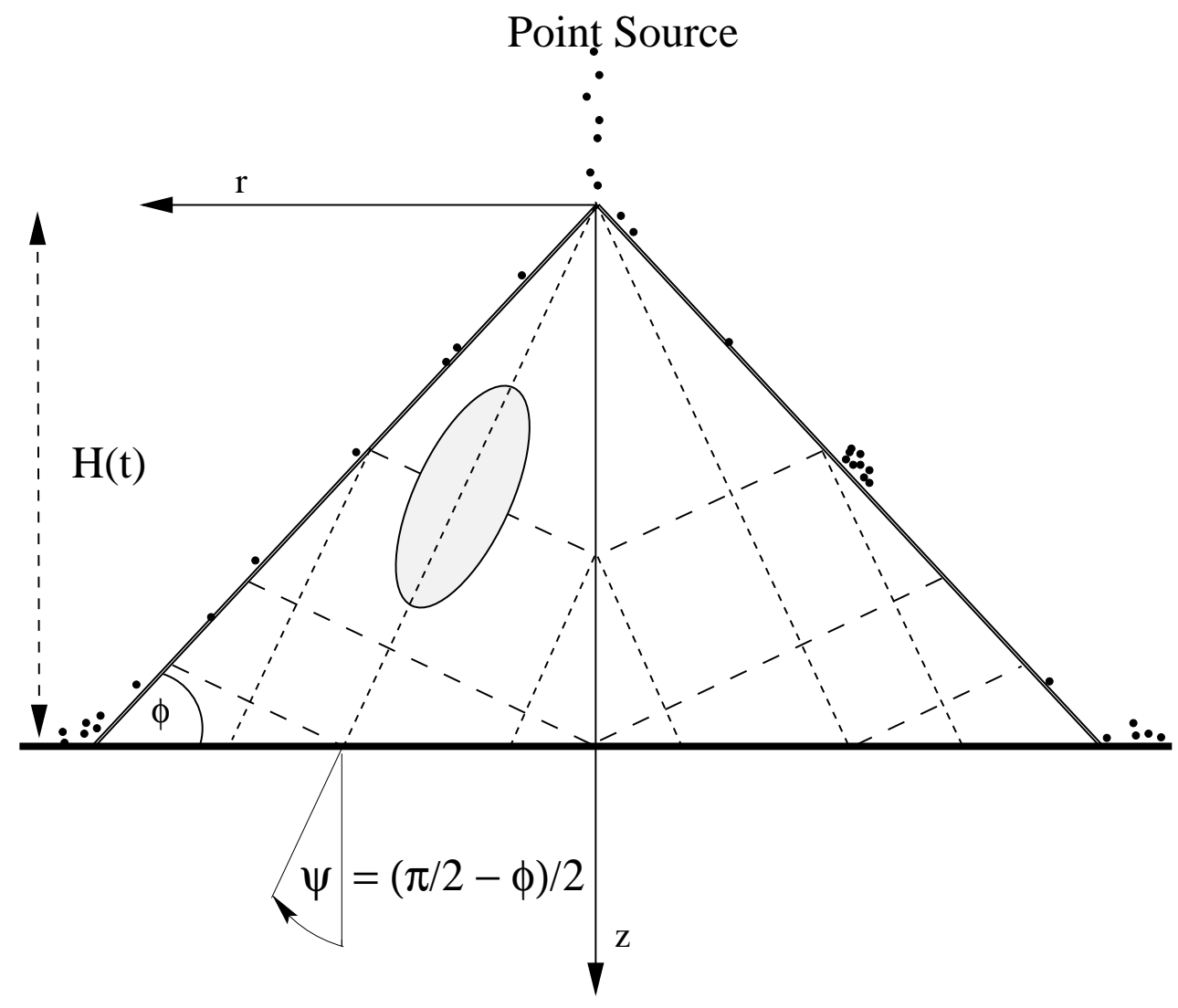

FIG. 5. Definition of normal construction history of a pile. The grains fall down from the point source on the pile and roll down the slopes, which are at the repose angle $\phi$. The height of this pile is $H(t)$. Sketch of the geometry of the FPA model: The stress ellipsoid has fixed inclination angle $\Psi$; its ellipticity varies from zero at the centre of the pile to a maximum in the outer region. The outward and inward stress propagation characteristics are indicated by short-dashed and long-dashed lines; these are at rightangles and coincident with the principal axed of the stress ellipsoid. 


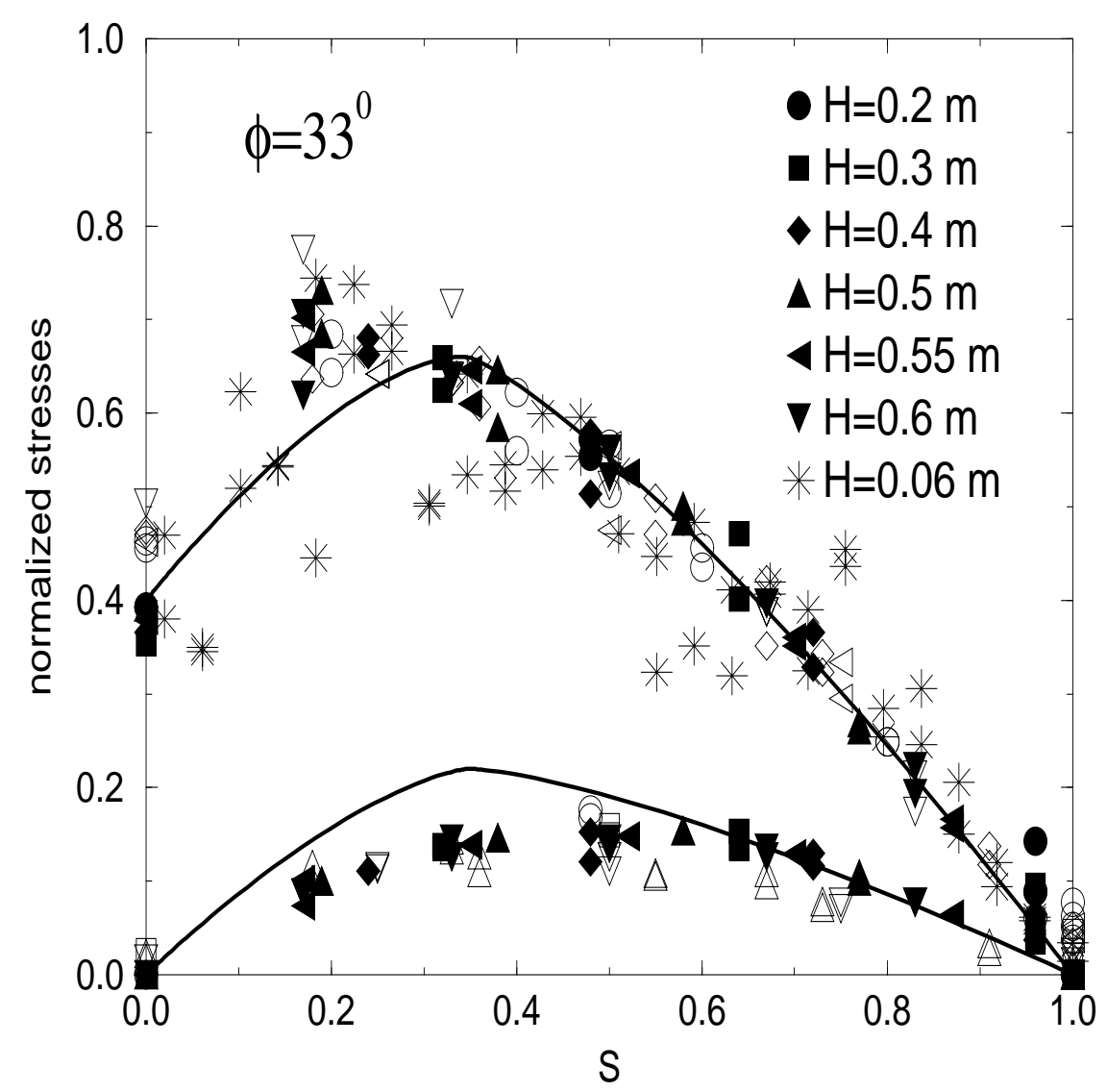

FIG. 6. Comparison of FPA model Using a uniaxial secondary closure [8, 9] with scaled experimental data of Smid \& Novosad [32] and $(*)$ that of Brockbank et al. [33] which was averaged over three piles. Upper and lower curves denote normal and shear stresses. The data is used to calculate the total weight of the pile which is then used as a scale factor for stresses. The horizontal coordinate $S=r \tan (\phi) / H$ is scaled by the pile height $H$. 
(a)

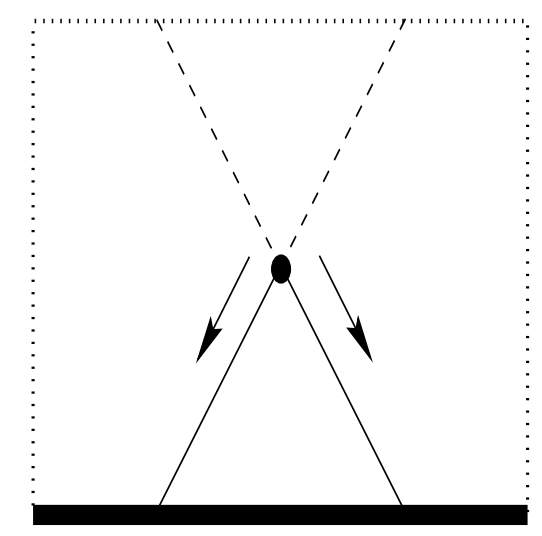

(b)

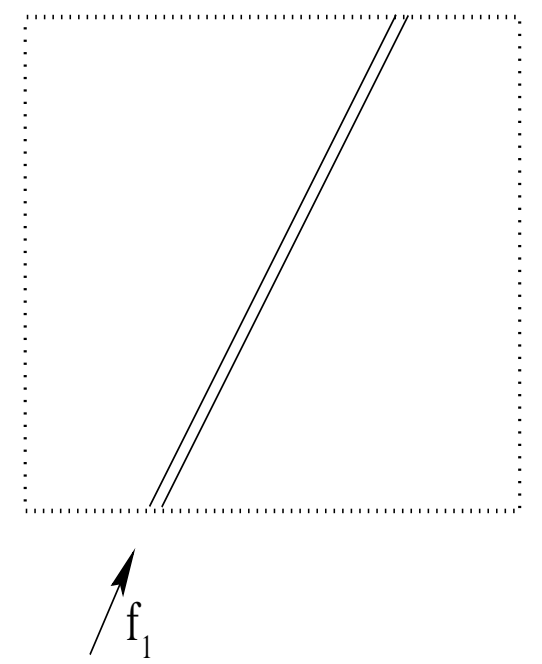

FIG. 7. (a) The response to a localized force is found by resolving it along characteristics through the point of application, propagating along those which do not cut a surface on which the relevant force component is specified. For a pile under gravity, propagation is only along the downward rays. (b) Admissible boundary conditions cannot specify separately the force component at both ends of the same characteristic. If these forces are unbalanced (after allowing for body forces), static equilibrium is lost. 

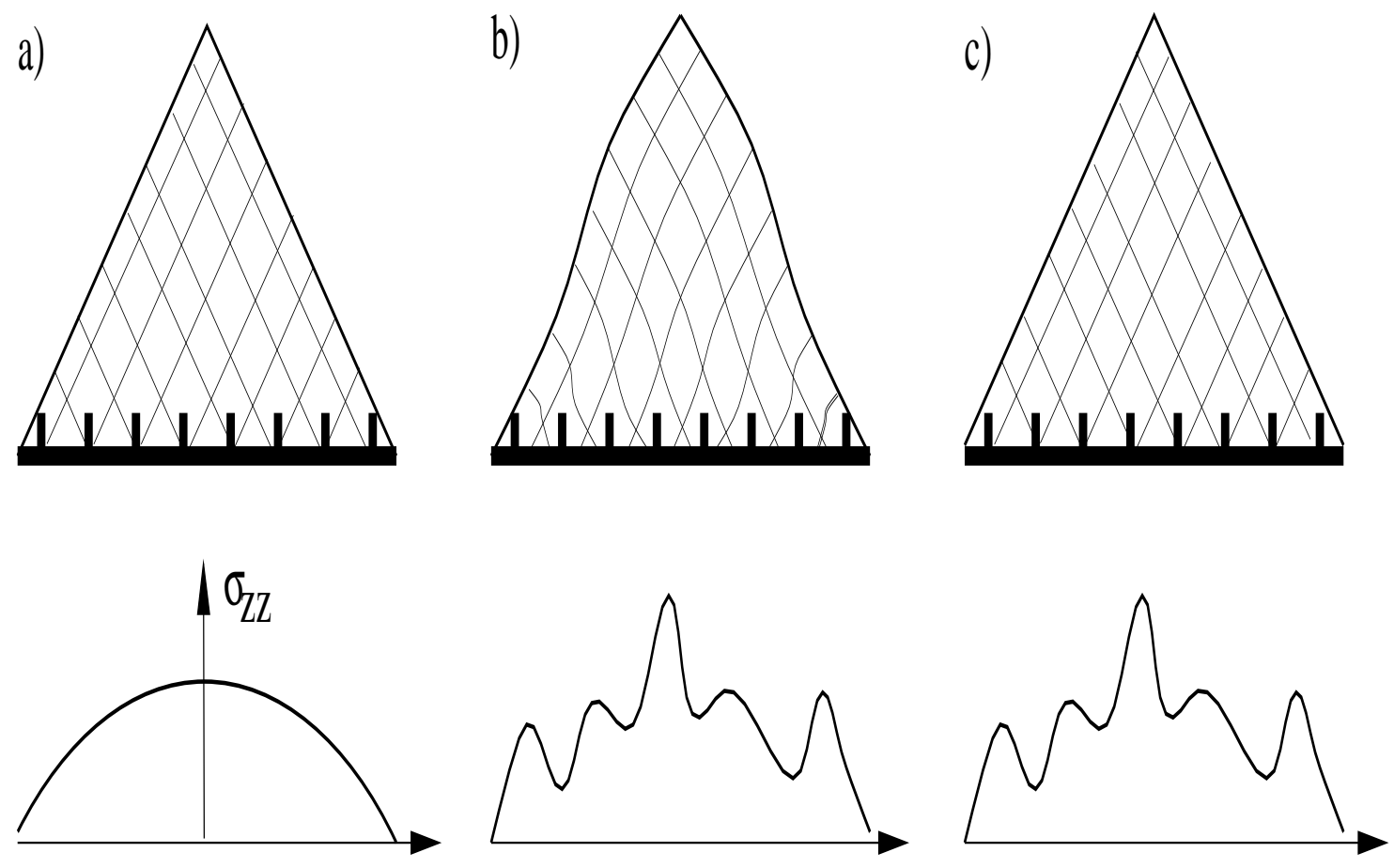

X

FIG. 8. Starting from an elastic cone or wedge on a rough support, any initial stress distribution can be converted to another by displacements with respect to the rough 'pinning' surface (a) $\rightarrow$ (b). Taking the limit of a high modulus $(\mathrm{b}) \rightarrow(\mathrm{c})$ at fixed surface forces, an arbitrary stress field remains, while recovering the initial shape of the cone and satisfying the free surface boundary conditions. This shows the physical character of 'elastic indeterminacy' for an elastic or elastoplastic body on a rough support. 


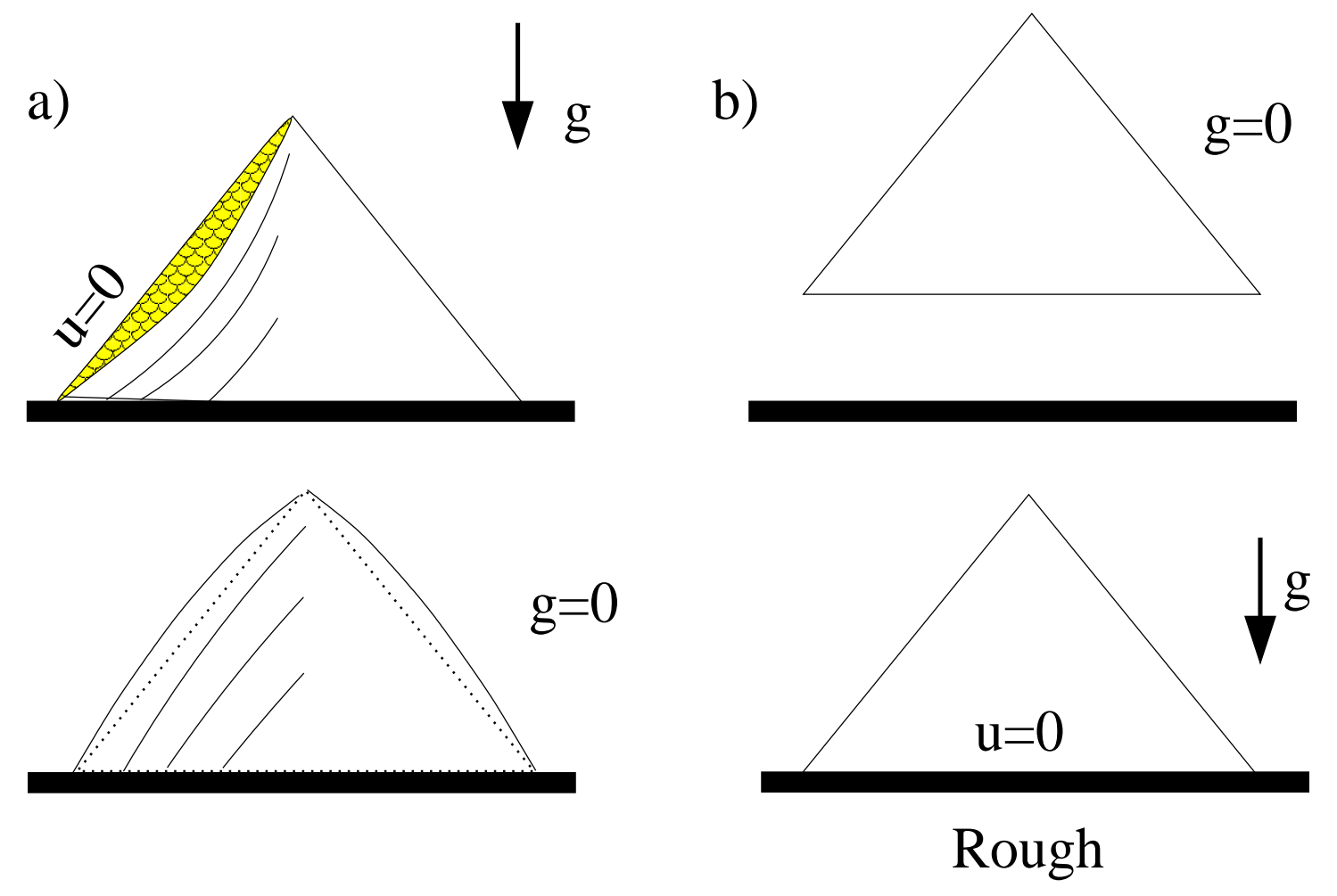

FIG. 9. (a) Quenched stresses in an elastic sandpile. Layers are added in a state of zero stress to a pre-existing, gravitationally loaded pile. Such a pile (if gravity is removed) will spring into a new shape, characterized by a nonzero internal stress field which includes tensile streses. These require rearrangements if the grains are cohesionless; the response to gravity is intrinsically nonlinear. (b) The 'floating' model of a sandpile. An unstrained, isotropic elastoplastic cone is brought into contact with a rough surface and gravity then switched on. This is the only construction history we can think of that completely avoids quenched stresses in the formation of the pile. 


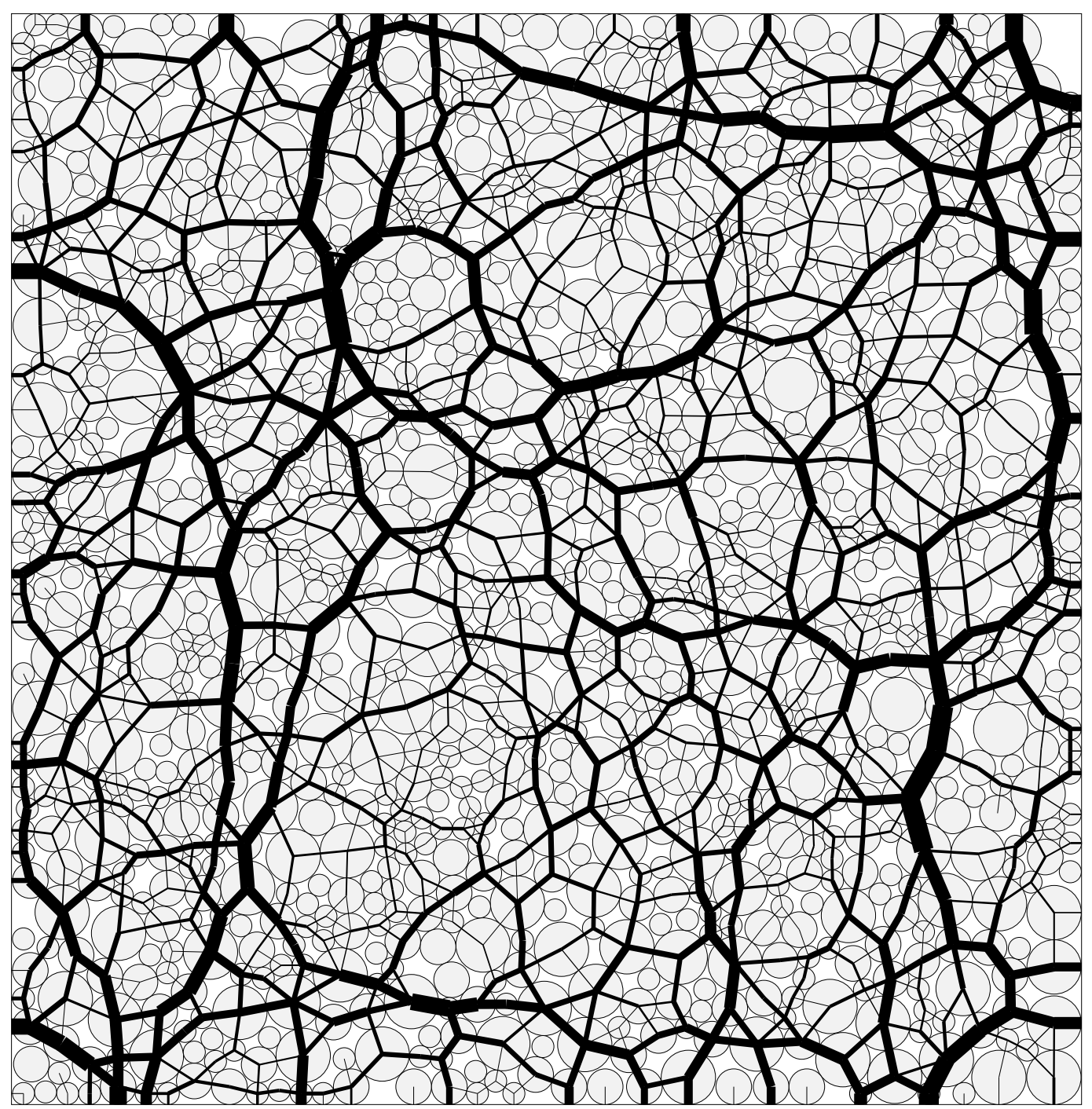




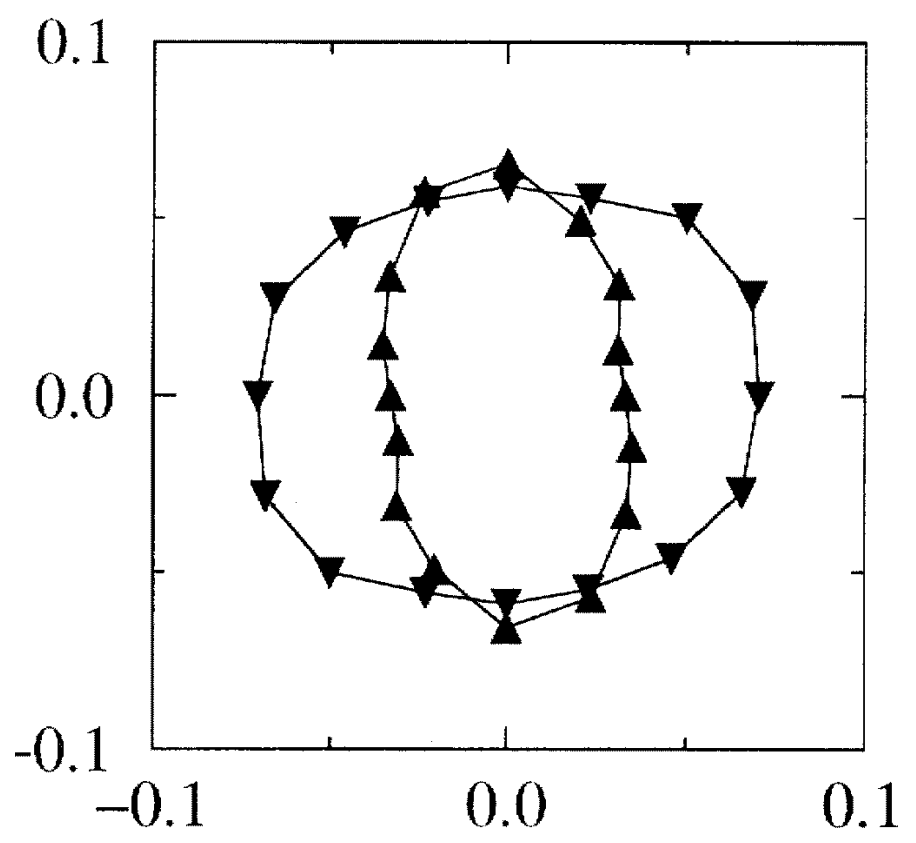

